\title{
PeUCESTA, TRA LEALISMo MACEDONE E MODELlo PERSIANO
}

\author{
Federicomaria Muccioli
}

Università di Bologna

\begin{abstract}
The paper aims to provide an overview of the career and figure of Peucestas, close friend of Alexander the Great and satrap of Persis from 326/5 until the final struggle against Antigonus the One-Eyed. Special attention is devoted to the ceremony of Persepolis (317 or 316 BCE) in the more general context of Persian patterns and Greco-Macedonian rituals and ceremonies.
\end{abstract}

Key words: Peucestas, Eumenes, Alexander the Great, Hellenism, ruler-cult, Hellenicity, intercultural changes.

La figura di Peucesta ha sempre suscitato un'attenzione limitata presso gli studiosi, se si escludono alcune importanti eccezioni. ${ }^{1}$ Educato insieme ad Alessandro a Mieza, probabilmente dallo stesso Aristotele, e al fianco del Macedone nella campagna asiatica cone ipaspista, divenne un protagonista nel 326/5 (forse primi mesi del 325), all'epoca dell'assedio in India di una innominata città dei Malli. In quell'occasione, secondo la tradizione più attendibile, che risale già ad Aristobulo, salvò la vita, da solo o assieme ad altri, al figlio di Filippo. Un episodio che divenne oggetto di una rielaborazione e falsificazione, in cui la pubblicistica (in particolare, da Clitarco a Timagene) tende in modo interessato a sostituire il nome di Tolemeo a quello di Peucesta come principale salvatore di Alessandro e a trasferire erroneamente il quadro dello scontro tra gli Oxidrakai, altra tribù indiana. ${ }^{2}$ Una mistificazione pesante, che non tiene conto della testimonianza dello stesso Tolemeo, il quale afferma esplicitamente che egli non partecipò a quell'episodio, in quanto impegnato militarmente altrove. In questo contesto rientra, forse in un periodo assai tardo (con Timagene?), anche l'attribuzione

1 Fondamentale è Wiesehöfer 1994, 50-56 e passim (cfr. Wiesehöfer 2007, 38-39; Wiesehöfer 2010, passim). Cfr. comunque Berve 1926, II: 318-319, no. 634 (e Berve 1926, I: 262-263 e passim); Heckel 1992, 263-267; Heckel 2006, 203-205; Billows 1990, 417-418 e passim. Tutte le date vanno intese a.C.

2 Vd. Plut. Alex. 63; De Alex. Magni fort. aut virt. 1.327b; 2.341c, 343d-344a, 344c-345b; Diod. 17.98-99; Curt. Ruf. 9.4.15-5.30 (5. 21 = in cui sono contenuti Clit, FGrH/BNJ 137 F 24; Ptolem., FGrH 138 F 26b = Timag., FGrH/BNJ 88 F 3); Arr. Anab. 6.8-12, partic. 10-11 (11.2-8= Ptolem., FGrH 138 F 26a); Ep. Metz 75-77. Per una panoramica completa e un'analisi al riguardo cfr. Muccioli 2018 b (di cui il presente contributo costituisce una prosecuzione nella ricerca). 
dell'appellativo Soter al Lagide proprio in virtù del suo supposto intervento in favore di Alessandro. ${ }^{3}$

A ciò si aggiunga che Peucesta è considerato, implicitamente, uno degli autori della congiura contro il figlio di Filippo, quantunque solo nella tradizione più tarda. Infatti egli avrebbe partecipò al banchetto organizzato da Medeio a Babilonia in cui Alessandro cadde malato secondo il Romanzo di Alessandro. ${ }^{4} \mathrm{Nel}$ Liber de morte Alexandri Magni poi, testo in cui, secondo Bosworth, andrebbe individuata una precisa tendenza filotolemaica, non rientra tra i sei che furono ignari del complotto per avvelenare il Macedone (ovvero Eumene, Perdicca, Tolemeo, Lisimaco, Asandro, Olcia). ${ }^{5}$ D'altro canto, secondo Arriano, egli fu tra coloro che consultarono Serapide sulla salute del re, passo peraltro quanto mai sospetto per il problema dell' esistenza della divinità già in quel periodo. ${ }^{6}$

$\grave{E}$ in atto dunque la tendenza a tacerne il ruolo al fianco di Alessandro e a ridimensionarne l'importanza, già a partire dal primo Ellenismo (ammesso che Clitarco risalga a quel periodo, all'epoca di Tolemeo II o già di Tolemeo I, nonostante testimonianze papiracee dissonanti $)^{7}$. Una tendenza che affiora tanto nelle fonti filolagidi, riguardo al primo punto, quanto in quelle legate ad Eumene e agli Antigonidi, come si vedrà.

In realtà il ruolo decisivo di Peucesta nello scontro con i Malli è riconosciuto dallo stesso Alessandro: secondo il racconto di Arriano (che attinge ad Aristobulo), questi in Carmania nel 325 ammise l'amico tra i somatophylakes, prologo alla concessione della satrapia di Perside. ${ }^{8}$ Successivamente, lo gratificò di una corona d'oro a Susa nel 324, per averlo difeso con lo scudo contro i Malli, così come fece anche con Leonnato (che intervenne anch'egli in quell'occasione). ${ }^{9}$

Particolare importanza riveste l'assegnazione della Perside, nel quadro di un'analisi più ampia, che riguarda i rapporti tra Macedoni e cultura iranica, durante la spedizione del 334-323 e successivamente, negli scontri per la conquista dell'egemonia. Peucesta infatti ebbe quell'incarico perché era particolarmente apprezzato da Alessandro per il

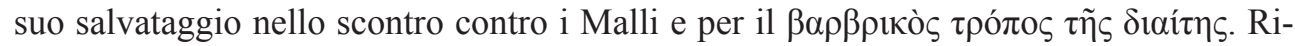
pagandolo della fiducia, egli, appena ottenuta la satrapia, adottò, unico tra i Macedoni, la veste meda; recepì i costumi persiani, imparandone anche la lingua. ${ }^{10}$ Una duttilità e un'apertura al mondo iranico mal tollerate dai compagni di Alessandro, che temevano l'imbarbarimento del loro leader. ${ }^{11}$

La satrapia della Perside fino a quel momento era riservata ad Iranici. Dapprima era stata appannaggio di Phrasaortes, alla cui morte si insediò, in modo del tutto illegale, Orxines. Questi venne condannato a morte e la regione passò così definitivamente a Peucesta. ${ }^{12}$ Va sostanzialmente condivisa l'interpretazione di Bosworth, che sottolinea

3 Arr. Anab. 6.11.8.

4 Ps. Callisth. 3.31.8. Cfr. Heckel 1988, 38-39, 74-75; Heckel 1992, 204.

512 (98). Cfr. Bosworth 2002, 126, nota 109.

6 Arr. Anab. 7.26.2.

${ }^{7}$ Cfr. lo status quaestionis di L. Prandi, in FGrH/BNJ 137 T1b (sul problematico P. Oxy. 71.4808, che testimonierebbe un rapporto di Clitarco con Tolemeo IV).

${ }^{8}$ Arr. Anab. 6.28.3-4 (=Aristob., FGrH/BNJ 139 F 50) e 30.2-3; cfr. Diod. 19.14.5.

9 Arr. Anab. 7.5.4-5.

${ }_{10}$ Arr. Anab. 6.30.3.

11 Arr. Anab. 7.6.3.

12 Arr. Anab. 6.30. 
come tale scelta di Alessandro costituisca un esempio di Realpolitik. ${ }^{13}$ Una scelta fortunata, che portò tra l'altro ad un incremento del contingente iranico nell'esercito del figlio di Filippo. Infatti a Babilonia, nel 323, Alessandro provvide ad una riorganizzazione del suo schieramento, con l'inserimento di molti Persiani nella falange macedone, dopo che Peucesta gli si presentò a lui, tornato dalla Perside con un esercito di ventimila Persiani, che comprendeva anche molti Cossei e Tapuri (tribù tra le più bellicose). ${ }^{14}$

La ricezione dei costumi persiani e la comunicazione linguistica, senza l'ausilio di un interprete, ${ }^{15}$ non sono in assoluto un unicum nel mondo greco, e riguardano anche figure di spessore come Temistocle ed Alcibiade. ${ }^{16}$ Per 1'epoca di Alessandro, invece, non abbiamo attestazioni di altri casi di esplicita volontà di assimilazione non solo della lingua ma anche della cultura iranica. ${ }^{17}$

A parte il caso di Peucesta, si assiste ad un diaframma che è linguistico, ma che è anche, in buona misura, frutto di una diffidenza dei Macedoni più ampia e di un'impermeabilità culturale, lato sensu. Questa è simboleggiata dal rifiuto di accettare la proskynesis: un rituale di corte, frainteso nel suo esatto significato e che si volle caricare eccessivamente di una valenza religiosa. ${ }^{18}$

L'atteggiamento dei diadochi nei confronti dei due poli - modello macedone e modello iranico - prima facie sembra essere stato decisamente sbilanciato a favore del primo, con un'attrazione fortissima per la regione di provenienza (si pensi, ad es., a Seleuco e al suo $\pi$ ó 0 os della patria, la Macedonia, ancora nel 281). ${ }^{19} \grave{E}$ comunque bilanciato da altri elementi, che indicano una certa apertura nei confronti del mondo iranico $\mathrm{e}$, in generale, la capacità di relazionarsi con realtà epicorie e le loro élites (a Babilonia come nel mondo iranico) senza peraltro perdere i tratti costituitivi della monarchia e 'demacedonizzarsi'. ${ }^{20}$ Questo vale soprattutto per lo stesso Seleuco ma anche per diversi suoi successori e, in parte, anche per gli usurpatori. ${ }^{21}$

A tal proposito si ricorda spesso che Seleuco fu l'unico tra i diadochi a non ripudiare la moglie di origine iranica, sposata a Susa nel 324, il che costituirebbe la prova che gli altri compagni di Alessandro abbandonarono ben presto il tentativo di fusione tra

13 Bosworth 1980, 12: "Doubtless Alexander had laid to heart the lesson of Orxines' usurpation and concluded that in Persis his satrap had to conform and be seen to conform to the local mores". Cfr. anche Hamilton 1987. Le motivazioni del Macedone sono ben colte da Diod. 19.14.5, dove, ripercorrendo brevemente le vicende di Peucesta e il suo rapporto con Alessandro, si sostiene che il suo uso della veste persiana in Perside era solo strumentale, per ottenere un'assoluta sottomissione degli epicori.

14 Arr. Anab. 7.23.1 e 3-5 (anche sull'elogio di Alessandro a Peucesta); cfr. 7.6.3; Diod. 17.110.2 (con probabile errata datazione dell'episodio a Susa nel 324; cfr. A. Zambrini, in Sisti/Zambrini 2004, 642, 644645; Prandi 2013, 188-189, per un tentativo di armonizzare le fonti); 19.14.5.

15 Cfr. Schirripa 2015 (con la principale bibliografia precedente).

16 Riferimenti in Muccioli 2013, 326-327, nota 210. Più in generale cfr. Hall 2002; De Luna 2003.

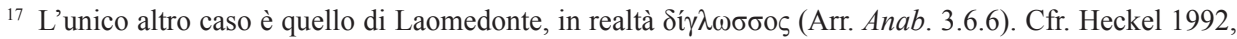
81-82, 190-193, 195-196; Heckel 2006, 146.

18 Cfr. Muccioli 2016.

19 Così emerge da Phot. Bibl. 224.226a, 1. 40 Henry (= Memn., FGrH/BNJ 434 F 8.1); vd. anche Paus. 1.16.2; App. Syr. 62.328 ss. Cfr. Muccioli 2004, 139-140.

$20 \mathrm{Su}$ questi aspetti vd. la discussione critica proposta in Muccioli 2017.

${ }^{21}$ Cfr. Chrubasik 2016. Vd. però la ribellione di Timarco nel II secolo, che taluni hanno voluto ritenere, non senza enfatizzazioni, l' 'erede' della politica iranica di Peucesta. Così Hoover 2011, 212 e nota 75, che si sofferma anche su alcuni simboli ritenuti validi per il mondo iranico nella monetazione di Seleuco I. 
elemento macedone ed elemento iranico, ai più alti livelli, a favore di una politica matrimoniale basata sulle relazioni tra i diadochi. L'affermazione è vera solo in rapporto al vacuum nelle nostre conoscenze, come ha recentemente rimarcato Meeus (comunque sia, nessuno dei principali protagonisti del primo Ellenismo coltivò matrimoni misti di questo tipo, da Antigono a Tolemeo). ${ }^{22}$ Sia pure seguendo un argumentum ex silentio, si deve quindi supporre che lo stesso Peucesta abbia sposato una donna iranica e che nessun motivo politico abbia causato un eventuale ripudio dopo la morte di Alessandro. ${ }^{23}$

E purtuttavia, seguendo questo filone d'indagine, non si può dimenticare come molti degli uomini vicini ad Alessandro, oltre a Peucesta abbiano recepito alcuni aspetti persiani, in modo giudicato negativamente dalle fonti, anche quelle coeve. Esaminando infatti la tradizione sul comportamento degli uomini vicini ad Alessandro, si nota una critica costante al culto della personalità (preludio ad un vero e proprio culto del sovrano), di cui un elemento costante è costituito dall'apertura a pratiche e costumi tipicamente persiani.

Così è, in particolare, per Arpalo, secondo il duro giudizio di Teopompo di Chio, con rimando alla proskynesis per la sua amante Glicera. ${ }^{24}$

Plutarco scrive che Clito, con ogni evidenza dopo la battaglia di Amorgo, si fece proclamare Poseidone ed era solito portare il tridente. ${ }^{25}$ Il luogo, per quanto inserito in un contesto fortemente sarcastico, va confrontato con altri, tendenti a sottolineare il protagonismo del personaggio. In un passo di Eliano, che ha riscontri in Ateneo e, in ultima analisi, risale a Filarco e Agatarchide, sono stigmatizzati i comportamenti, tendenti al lusso e alla dissipatezza, di Agnone, Perdicca, Cratero, Leonnato e Menelao, oltre che di Clito; in particolare, di quest'ultimo si afferma che «quando doveva ricevere qualcuno, dava udienza camminando su tappeti di porpora». ${ }^{26}$

Tale tendenza denigratoria non è comune solo a Filarco e ad Agatarchide (e alla tradizione che da loro dipende) ma trova un pendant in Duride di Samo, pronto a criticare il lusso dei diadochi (oltre ad avere parole dure nei confronti del culto del sovrano e di quelle che sono considerate forme di adulazione).$^{27}$

Per quanto riguarda il lusso (ispirato, più o meno direttamente, all'esperienza persiana), spicca un luogo dell'opera Sullo stile, attribuita a Demetrio. Qui Demetrio Falereo biasima, ricorrendo al discorso figurato, l'altezzoso orgoglio del macedone Cratero, che riceveva le ambascerie greche assiso su una kline d'oro, con indosso un mantello color porpora. ${ }^{28}$

Dal canto suo, Arriano recepisce la tradizione storiografica del primo Ellenismo, nella sua opera Gli avvenimenti dopo Alessandro, come si può desumere da alcuni lemmi della Suda. In particolare, si sofferma su alcuni tratti caratteriali ed esteriori di Cratero, sottolineando gli aspetti imitativi nei confronti di Alessandro Magno, peraltro in un contesto

${ }^{22}$ Meeus 2009, 236; Meeus 2013, 88-89.

${ }^{23}$ Il confronto tra Peucesta e Seleuco è anche in Brosius 2003, 177-178, secondo la quale Peucesta avrebbe sposato una donna persiana. Cfr. Briant 2008, 210.

${ }^{24}$ Athen. 13.595d; cf. 13.586c-d (= Theop., FGrH/BNJ 115 F 254b; cfr. FF 253, 254a, riguardo agli onori per la precedente amante Pizionice). Cfr. Muccioli 2016, 45 e nota 31.

${ }_{25}$ Plut. De Alex. Magni fort. aut virt. 2.338a.

${ }_{26}$ Ael. V.H. 9.3; cfr. Athen. 12.539b-c (= Phylarch., FGrH 81 F 41 = Agatharch., FGrH 86 F 3).

${ }^{27}$ Passi e analisi in Muccioli 2018a; cfr. anche, più in generale, Pownall 2013.

${ }^{28}$ Demetr. De elocut. 289 (= F 12 Stork - van Ophuijsen - Dorandi). 
altamente positivo. ${ }^{29}$ Critica invece le aperture alle pratiche persiane di un personaggio come l'ambizioso Leonnato, che nel 323 ebbe il controllo della Frigia Ellespontica, trovando poi la morte nella guerra lamiaca. Costui, alla morte di Alessandro, si dimostrò sensibile sia al modello del Macedone sia al lusso persiano nelle vesti e nell'acconciatura

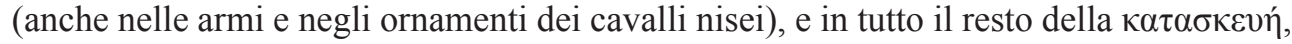

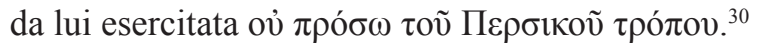

Vi fu dunque chi tra i diadochi fu attratto da una rappresentazione del potere che, agli occhi dei contemporanei, creava pericolose commistioni con l'idea di regalità tipica del mondo persiano, il che si riflette in una valutazione negativa della tradizione, arrivata fino ad Arriano. Tale (pre)giudizio riguarda i personaggi in questione, ma va esteso alla più generale interpretazione del mondo persiano come animato solo dalla $\tau \rho v \varphi \eta ́$, autentica chiave di lettura trasversale, dall'età arcaica alla fine dell'impero achemenide (e considerata anche motivo ultimo della crisi del IV secolo). ${ }^{31}$

Tornando più propriamente a Peucesta, il suo ruolo di satrapo della regione venne confermato a Babilonia nel 323, dopo la morte di Alessandro ${ }^{32}$ e successivamente a Triparadiso (nel 321 o 320). ${ }^{33}$ il suo rapporto con la Perside, se da un lato lo pose in una posizione privilegiata, dall'altra fu una camicia di Nesso, finendo per relegarlo geograficamente e politicamente.

La tradizione della e sulla prima età ellenistica è incapace di offrire culturalmente un'immagine esatta di questa figura, per motivi in primo luogo di opportunità politica (in particolare il rapporto di Ieronimo di Cardia con Eumene e poi con gli Antigonidi e fonte fondamentale a riguardo) o anche per mancata finezza interpretativa. Non sorprende dunque la sua rappresentazione negativa nella Vita di Eumene di Plutarco e in alcuni passi della Biblioteca storica di Diodoro Siculo.

Senza inoltrarsi nella complessa questione della Quellenforschung diodorea, è pressoché concordemente riconosciuto che la fonte (ultima) dello storico di Agirio nei luoghi che riguardano Peucesta sia, in buona sostanza, Ieronimo di Cardia. Questi fu presumibilmente anche fonte oculare, vista la dettagliata descrizione di luoghi e avvenimenti nel libro XIX. ${ }^{34}$ Più complesso il quadro nella Vita di Eumene, che coinvolge anche i criteri compositivi e le motivazioni sottese nella scelta del personaggio. Può senz'altro sembrare sorprendente la scelta plutarchea di scrivere una biografia di Eumene, personaggio assai sfocato e minore rispetto ad altri del periodo, tralasciando invece figure di maggior spicco (come Seleuco I o Tolemeo I). Bsogna probabilmente ritenere che l'esempio stesso di Nepote, autore di una biografia del personaggio, abbia influito nella decisione di cimentarsi sulle vicende di Eumene. Ma non va trascurata anche la possibilità che adeguato materiale di tipo biografico fosse reperibile nelle opere preeesistenti e facilmente consultabile, come appunto Ieronimo o anche Duride di

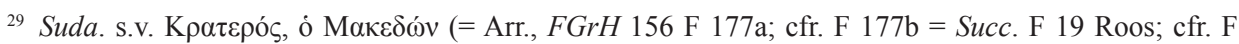
26 Roos).

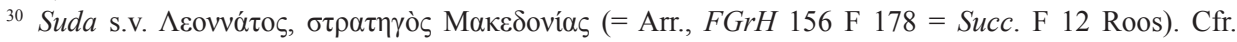
Bosworth 2002, 58, nota 108 (che accentua solo l'aspetto dell'imitatio Alexandri).

${ }^{31}$ Cfr. Bernhardt 2003, 239-247 e, specificatamente sul tema della decadenza persiana, Briant 1989.

${ }^{32}$ Diod. 18.3.3; Iust. 13.4.23; Dexipp., FGrH 100 F 8, 6; cfr. Liber de morte 121.

${ }_{33}$ Diod. 18.39.6; Phot. Bibl. 92.71b, 1l. 31-32 Henry (= Arr., FGrH 156 F $9.35=$ Succ. F 1.35 Roos).

${ }^{34}$ Ieronimo come fonte guida è ipotesi di Hornblower 1981. Per un uso combinato si pronuncia Landucci Gattinoni 2008. L'ipotesi della Mittelquelle è ora avanzata da Rathmann 2014; Rathmann 2016. 
Samo (la cui tendenza nei confronti di Eumene non è peraltro facilmente evincibile). ${ }^{35}$ Non ha riscosso grande fortuna l'ipotesi sostenuta da Hadley, sull'esistenza di una fonte (peraltro non meglio specificabile) favorevole ad Eumene e a cui risalirebbe la tradizione successiva. ${ }^{36}$

Se dunque, complessivamente, è tangibile la traccia di Ieronimo, è ben comprensibile che costui abbia messo in cattiva luce tanto Peucesta quanto i satrapi ai lui legati. ${ }^{37}$ A Peucesta viene negato ogni spessore militare (fondamentale nella valutazione dell'areté dei diadochi), in quanto viene presentato come litigioso e codardo. ${ }^{38}$ In particolare, Plutarco (che comprime eccessivamente gli avvenimenti nella sua narrazione) appiattisce il suo ruolo su quello dei satrapi anellenici a lui alleati, con la riproposizione di topoi logori, ma comunque pur sempre efficaci agli occhi dei lettori: l'arroganza barbarica, la violenza, la ricchezza dissoluta che ha il solo risultato di corrompere l'accampamento e l'esercito. ${ }^{39}$

Riguardo all'attività di Peucesta negli anni degli scontri tra i diadochi, oggetto di ampio dibattito critico e che qui non si vuole ripercorrere nelle sue linee evenemenziali, spicca la $\theta v \sigma i ́ \alpha \mu \varepsilon \gamma \alpha \lambda \circ \pi \rho \varepsilon \pi \eta ́ \varsigma$ di Persepoli, già capitale dinastica degli Achemenidi e città cardine della satrapia di Perside, e datata al $317 \mathrm{o}$ al $316,{ }^{40}$ in occasione dell'incontro con Eumene. Questa è descritta nel dettaglio da Diodoro, ${ }^{41}$ mentre è solo accennata da

35 L'unico frammento riferito alle origini di Eumene e della sua famiglia è chiaramente tendenzioso, e non è sufficiente indizio di una metabolé del personaggio nella rappresentazione dello storico samio (Plut. Eum. 1.1-3 = FGrH/BNJ 76 F 53); cfr. Pownall 2013, 51.

${ }^{36}$ Hadley 2001, su cui cfr. Landucci Gattinoni 2004, 337, nota 14. Per altre osservazioni sulla scelta del personaggio, cfr. Bosworth 1992; Geiger 1995; Landucci Gattinoni 2004, 333-335; Muccioli 2012, 215-216, $232-233$.

37 Hornblower 1981, 151; Schäfer 2002, 156; Roisman 2010a, 137; Roisman 2010b, 73 (entrambi i contributi rifluiti in Roisman 2012, partic. 9 ss., 212 ss.); Anson 2015, 9.

38 Vd. i passi raccolti da Anson 2015, 5; cfr. Bosworth 1992, 68.

39 Eum. 13.9-11. Cfr. Landucci Gattinoni 2004, 443, nota 86 (per l'imprecisione cronologica).

40 Determinante è stabilire con precisione l'anno della morte di Eumene, fissata ora al 316 o al 315. È comunque difficile decidere tra cronologia alta e cronologia bassa; status quaestionis in Landucci Gattinoni 2011, che propende per quella bassa (cfr. Landucci Gattinoni 2004, 375-381, 383-384).

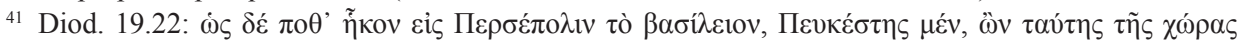

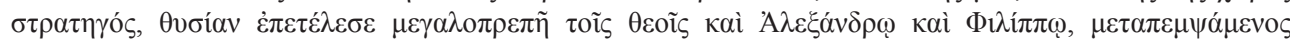

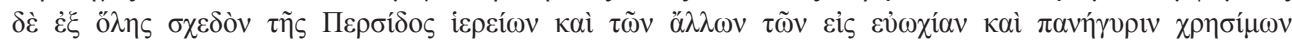

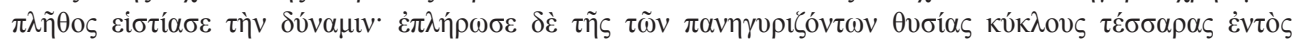

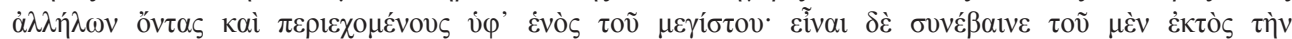

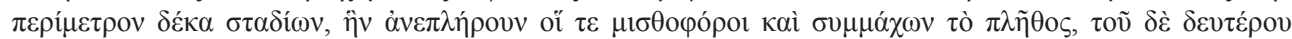

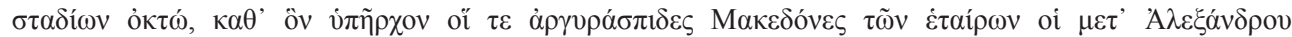

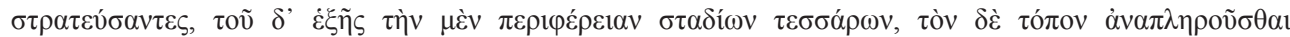

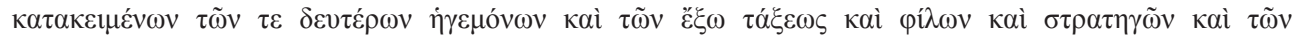

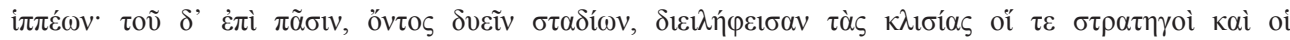

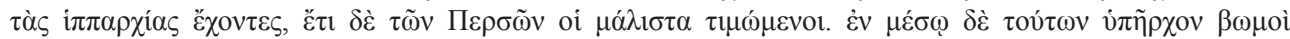

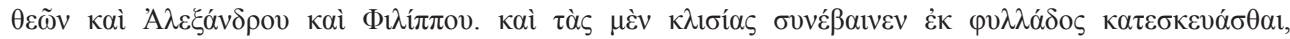

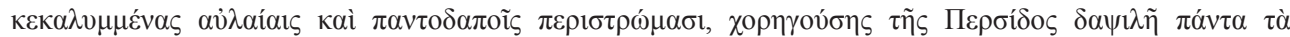

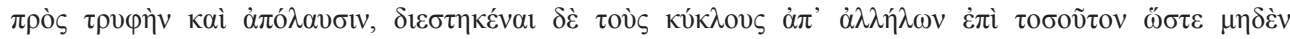

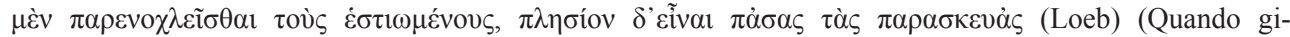
unsero a Persepoli, la capitale reale, Peucesta, stratego di questa regione, celebrò grandiosi sacrifici agli dèi, ad Alessandro e a Filippo, e, avendo fatto giungere da quasi tutta la Perside una gran quantità di vittime e di quanto altro era utile per il banchetto e la festa religiosa, invitò tutti i soldati. Dispose i partecipanti al 
Plutarco. ${ }^{42}$ Entrambe le fonti sottolineano comunque la volontà del satrapo (definito stratego della regione, in Diodoro) di attrarre dalla sua parte l'elemento militare, con il chiaro intento di avere il comando generale. ${ }^{43}$

L'Agiriense espone subito dopo lo stratagemma messo in atto da Eumene attraverso l'invio di false lettere, scritte in aramaico ("caratteri siriaci"), e spedite ad Oronte, satrapo di Armenia e amico di Peucesta, in cui si esaltava il suo operato e si sosteneva che Olimpiade, dopo aver accolto il figlio di Alessandro, aveva recuperato il regno di Macedonia, vista la morte di Cassandro; si affermava inoltre che Poliperconte era passato in Asia contro Antigono, con il fior fiore dell'esercito e gli elefanti, e che era già in Cappadocia. Uno stratagemma, con notizie palesemente false, volto a screditare Peucesta e che valse ad Eumene il favore dell'esercito, convinto che egli, attraverso l'appoggio dei re, avesse ormai il potere assoluto, e il rispetto dei satrapi. ${ }^{44}$

Invece, Plutarco, che nel complesso comprime gli avvenimenti con diverse sfasature cronologiche, tralascia completamente questi avvenimenti e si sofferma sulla malattia di Eumene e il rapporto con Antigono. ${ }^{45}$

La cerimonia descritta da Diodoro deriva da una fonte molto dettagliata e precisa, anche nella descrizione geografica. Non rinunciando ad una venatura critica tipicamente antipersiana (la sottolineatura della $\tau \rho v \varphi \eta ́$ e dell' $\alpha \dot{\alpha} \delta \dot{\lambda} \alpha v \sigma 1 \zeta$ dispiegate in quel contesto) tutto è proiettato in una dimensione decisamente rituale-religiosa, sottolineata dall'uso

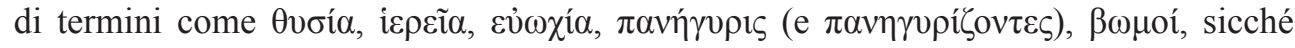
questa costituisce una chiave di lettura importante, ancorché non unica, per proporne l'esatta comprensione. Infatti la $\theta v \sigma i ́ \alpha$ nei confronti degli dèi, di Alessandro e Filippo viene spesso sottovalutata tra i classicisti, mentre da tempo ha attirato l'attenzione soprattutto degli iranisti e degli studiosi attenti al sostrato religioso e rituale. I primi hanno focalizzato l'attenzione soprattutto sulle sue finalità politiche, connettendola ai rapporti di Peucesta con Eumene (con Antigono e Seleuco sullo sfondo). Ragione per cui talora si è parlato addirittura di "extravant show of pomp", con chiaro fraintendimento. ${ }^{46}$

Purtuttavia la critica più attenta ha suggerito alcune possibilità di raffronto. Bosworth, in particolare, ha ritenuto che lo spunto per la disposizione concentrica dei partecipanti venga dalla cerimonia di Alessandro a Susa del 324, dove figura un trono d'oro al cen-

sacrificio in quattro cerchi concentrici e racchiusi da quello pì̀ grande; il perimetro di quello più esterno era di dieci stadi e lo occupavano i mercenari e la moltitudine degli alleati; quello del secondo cerchio era di otto stadi e vi si trovavano gli argiraspidi macedoni e gli hetairoi che avevano combattuto al seguito di Alessandro; il perimetro del successivo era di quattro stadi e vi avevano preso posto i comandanti in seconda, gli amici e gli strateghi fuori schiera e infine i cavalieri; nell'ultimo, che era di due stadi, sedevano gli strateghi, gli ipparchi e inoltre i più insigni Persiani. Nel mezzo c'erano gli altari degli dèi, di Alessandro e di Filippo. I sedili erano fatti di fogliame, ricoperti di tappeti e di ogni genere di tovaglie, dal momento che la Persia ha abbondanza di tutto quanto serve al lusso e al piacere; i cerchi infine erano collocati a una distanza tale l'uno dall'altro che i convitati non erano assolutamente a disagio e avevano vicino tutto quanto il necessario; trad. Simonetti Agostinetti con adattamenti).

${ }^{42}$ Eum. 14.5: Peucesta sperava di avere un ruolo di preminenza, in quanto in Perside aveva nutrito

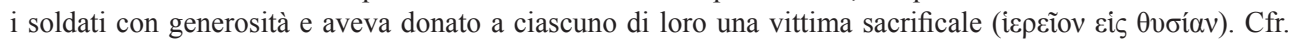
Polyaen. 4.8.3 (elargizioni di vino e distribuzioni di bottino ai soldati).

${ }^{43}$ Diod. 19. 23.1 (cfr. 21.3).

${ }^{44}$ Diod. 19.23. Cfr. Polyaen. 4.8.3.

${ }^{45}$ Eum. 14.6 ss.

${ }^{46}$ Heckel 1992, 245; Heckel 2006, 204. Cfr. Billows 1990, 417-418. 
tro. ${ }^{47}$ Dal canto suo, Tuplin considera possibile un collegamento con la $\theta v \sigma i ́ \alpha$ e la $\theta$ otvì $\delta \eta \mu о \tau \varepsilon \lambda \eta ́ \varsigma$ di Opis svoltasi nello stesso anno. Secondo Arriano, unica fonte in proposito, I Macedoni erano disposti intorno ad Alessandro, e di seguito, i Persiani e, dopo questi, altre genti, che si distinguevano per rango o per altre qualità. Magi e indovini greci diedero inizio alla cerimonia in cui tutti facevano le stesse libagioni e il figlio di Filippo pregava per l'armonia e la concordia tra Persiani e Macedoni. Il rito venne concluso con un peana intonato dai novemila partecipanti al rito. ${ }^{48}$ Decisamente forzato è invece il collegamento con quanto descritto da Polieno, sulla marcia di Antioco I verso Damasco,

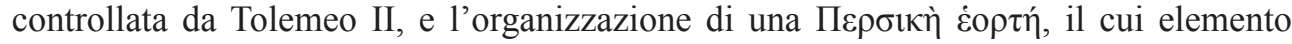

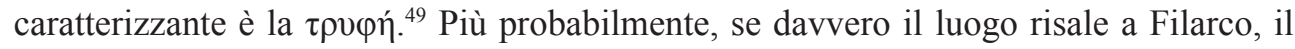
rimando al mondo persiano e al lusso va ridimensionato e inquadrato nel consueto topos della degenerazione orientale. ${ }^{50}$

Un altro confronto, e che anzi con più forza si impone, anche per la sua vicinanza temporale, è con quello con il cosiddetto culto del trono vuoto di Alessandro nel 318 o 317 a Cyinda in Cilicia, voluto da Eumene. Anzi, secondo Schäfer, la cerimonia di Peucesta sarebbe stata organizzata in una sorta di competizione proprio con quanto fatto da costui tempo prima. ${ }^{51}$ Diodoro, anche in questo caso la fonte più puntuale a riguardo, sottolineando l'emarginazione di Eumene rispetto ai Macedoni ai quali il potere spettava di diritto, ricorda quello che a tutti gli effetti si può considerare un suo stratagemma per recuperare prestigio e autorità e affermare il suo attaccamento alla memoria di Alessandro. Questi gli era infatti apparso in sogno, imponendogli di allestire un trono d'oro e ponendovi il diadema, lo scettro e le armi di cui si serviva; i generali avrebbero dovuto celebrare sacrifici e riunirsi in assemblea presso il trono, ricevendo gli ordini in nome del re, come se fosse ancora vivo. Eseguito l'ordine e preparato il trono dentro una magnifica tenda, i generali celebravano sacrifici su un altare con il fuoco, spandendo incenso e altri preziosissimi

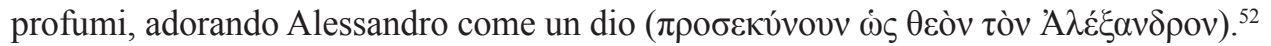

Il ricorso ad una vera proskynesis in quella circostanza è fortemente dubbio o, quanto meno, l'espressione è imprecisa; si può invece ammettere, con Launey e Picard, un culto militare per Alessandro, sorto in un milieu ben preciso, quello macedone (come dimostra anche la proposta di Tolemeo, a Babilonia nel 323, poco dopo la morte di Alessandro, che i compagni del re prendessero decisioni nell'interesse comune davanti al trono vuoto del Macedone nel padiglione reale, episodio con cui talora viene confrontato quello di Cyinda). ${ }^{53}$ Anzi, giustamente Anson considera quanto messo in atto da Eumene "the only

47 Athen. 12.539e-f (= Phylarch., FGrH 81 F 41); Ael. V.H. 9.3; Polyaen. 4.3.24. Cfr. Bosworth 2002, 121, nota 92; 255-256, seguito da Meeus 2009, 244.

48 Arr. Anab. 7.11.8-9. Cfr. Tuplin 2009, 129, nota 126.

49 4.15. Cfr. Kosmin 2014, 161 e nota 92.

50 Opportunamente Callieri 2014, 89, osserva che la festa riguarda (o meglio riguarderebbe: nel testo non se ne parla) l'elemento militare persiano, costretto ad arruolarsi.

51 Schäfer 2002, 141-143, sostanzialmente seguito da Anson 2015, 167, nota 8.

${ }^{52}$ Diod. 18.60.3-61.1 (cfr. 19.15.3-4); Polyaen. 4.8.2; Nep. Eum. 7.2; Plut. Eum. 13.8 (cfr. 2.10, dove, in un contesto tendenzioso e stonato rispetto al resto della biografia, si parla delle attenzioni del Cardiano per il culto eroico di Efestione). Eumene non è il solo ad avere la visione in sogno di Alessandro (peraltro ripetuta: Plut. Eum. 6.8-10): vd. i casi di Demetrio, Seleuco e Pirro (Plut. Demetr. 29.1-2; Diod. 19.90.3-4; Plut. Pyrrh. 11.4-5) su cui cfr. Meeus 2009, 244.

${ }_{53}$ Cfr. Launey 1950, 945-946, 951, seguito da Picard 1954, 5-6; vd. anche Muccioli 2016, 42 (con altra bibliografia). Riguardo a Tolemeo la sua proposta è nota solo da Curt. Ruf. 10.6.15: sede Alexandri in regia 
indisputable example of an essential military cult in the Hellenistic era". ${ }^{54}$ Infatti non vi sono elementi per sostenere, con Schäfer, che costituisse un tentativo di creare un culto divino di Alessandro, con una certa validità tra Macedoni, Greci e non Greci, giacché questi ultimi - lo si è detto - non sono minimamente menzionati. ${ }^{55}$

D'altro canto, la crìtica più attenta alla tradizione iranica ha sottolineato da tempo gli emprunts persiani nella $\theta v \sigma i ́ \alpha$ di Peucesta, con precise corrispondenze in rituali achemenidi (a cui si sarebbe uniformato anche Alessandro ad Opis). ${ }^{56}$ Come ha dimostrato in modo dettagliato Henkelman, un confronto serrato è proponibile con la cerimonia di Pasargade in onore di Dario I, organizzata dal dignitario achemenide Parnakka, nota dalle tavolette elamite. ${ }^{57}$ Utile è anche il confronto con il sacrificio per Zeus Stratios nell' 82 da parte di Mitridate VI del Ponto, così come è descritto da Appiano nel Libro mitridatico e che si richiama esplicitamente a quanto fatto a suo tempo dai re persiani a Pasargade. ${ }^{58}$

Vi è dunque da chiedersi se vi sia un diaframma interpretativo nella descrizione della cerimonia di Persepoli, a causa del fatto che questa è nota solo dalle fonti greche (e dunque, inevitabilmente, parziali se non incomplete). In altri termini, nella $\theta v \sigma i ́ \alpha$ gli elementi greco-macedoni sono preponderanti, a rischio di sbilanciare l'interpretazione in un senso ben preciso (nonostante si affermi che ai più insigni dei Persiani era riservata una posizione di prestigio nell'ultimo dei quattro cerchi, secondo una disposizione che risente più dell'esempio di Parnakka che di quelli di Alessandro, per non parlare della cerimonia di Eumene). ${ }^{59}$ Diodoro (attingendo a Ieronimo, probabile fonte diretta, come si è detto $)^{60}$ parla genericamente di altari per gli dèi, accostandoli a quelli per Alessandro e Filippo, suggerendo sia pur implicitamente al lettore l'idea che queste divinità fossero (sostanzialmente o formalmente?) greche, e che non dovessero esservi comprese anche quelle persiane. È poi ricordata la presenza di altari per Alessandro e Filippo, non già anche quelli per Ciro II o altri sovrani achemenidi.

La documentazione epigrafica fornisce al riguardo elementi importanti, ma non dirimenti. A Persepoli sono state rinvenute infatti cinque piccole tavole di calcare grigio da applicare probabilmente a degli altari, recanti ciascuna il nome di una divinità greca al genitivo (Zeus Megistos, Atena Basileia, Apollo, Artemide, Helios) senza alcun tentativo di interpretatio, come si registrerà successivamente, in Commagene con Antioco I. Sembra essere ormai prevalente l'ipotesi di collegare tali iscrizioni ad un periodo compreso tra la spedizione di Alessandro e l'epoca dei primi Seleucidi, mentre più aleatoria la possibilità che si riferiscano al periodo della dominazione dei frataraka

posita, ma è considerata generalmente attendibile. Cfr. Errington 1970, 75, seguito da Anson 2015, 167, secondo cui Eumene si sarebbe ispirato a questo precedente.

54 Anson 2015, 167. Sulla fine del culto con la morte di Eumene, cfr. Launey 1950, 947 (in cui la cerimonia di Peucesta è vista come una prosecuzione di quella di Cyinda).

55 Schäfer 2002, 21-37. Contra, Bosworth 2005, 685-686; Roisman 2010b, 78, nota 26.

56 Calmeyer 1982, 185-186; Wiesehöfer 1994, 53; Briant 2002, 247, 311, 766, 916; Henkelman 2011, 117.

57 Henkelman 2011, 115-118.

${ }^{58}$ Mithr., 66, 276-279; cfr. 70.295. Cfr. Ballesteros Pastor 2011 (con significativo cambiamento di interpretazione rispetto agli studi precedenti, tendenti a sottolineare soprattutto l'elemento greco), nonché, ad es. Calmeyer 1982, 185; Henkelman 2011, 89-90, 115; Eckhardt 2015, 282-286.

59 Opportunamente Calmeyer 1982, 185, osservava: "Schlachtopfer-Brand-Altäre wären recht uniranisch".

${ }^{60}$ Questo aspetto è sottolineato in Altheim/Stiehl 1970, 564-565. 
(variamente datata tra III e II secolo). ${ }^{61}$ Anzi, l'affermazione, spesso ripetuta, che siano state ritrovate nel locale tempio del fuoco è destituita di fondamento, il che inficia un rapporto diretto con la stessa dinastia dei frataraka ${ }^{62}$ Il richiamo ai primi Seleucidi, in particolare, è stato sostenuto soprattutto da Iossif e dalla Lorber che, riprendendo suggerimenti nella scholarship, propongono una interpretatio Graeca per Ahura Mazdā (Zeus Megistos), Anahita (Artemide e Atena Basileia) e Mithra (Helios). ${ }^{63}$ Questa però non ha precisi riscontri nella documentazione, mentre è più allettante collegare la datazione e l'interpretatio proprio con la cerimonia di Peucesta e gli altari per gli dèi lì disposti, secondo un'ipotesi di Wiesehöfer, più volte riproposta, che ha avuto un certo séguito. ${ }^{64}$

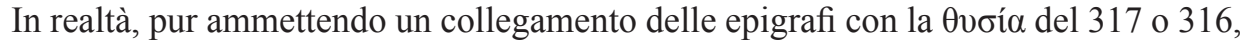
una precisa corrispondenza tra divinità greche e divinità persiane rimane aleatoria e conseguentemente va cautamente rifiutata. Se così è, dunque, questi documenti epigrafici costituiscono la controprova della predominanza del lato greco nella sfera sacrale-religiosa del rito di Persepoli, senza alcun tentativo diretto o comunque ostentato di omologare due mondi religiosi tra loro distanti.

Ciò detto, non si deve però affatto escludere la partecipazione attiva della componente persiana all'organizzazione della cerimonia. È vero che non vi sono elementi nelle fonti letterarie per sostenere una 'conversione' di Peucesta al Mazdeismo; ${ }^{65}$ tuttavia va anche adeguatamente tenuta in conto la sua grande popolarità presso il mondo persiano (che doveva essere trasversale, e comprendere sì le truppe ma anche le élites politiche e religiose), attestata già sotto Alessandro e mantenuta se non sviluppata negli anni tumultuosi degli scontri tra i diadochi. ${ }^{66}$ Significativa è la ribellione della componente iranica quando Antigono destituì Peucesta, dopo la vittoria su Eumene; questa si concluse con l'uccisione di Tespi, uno degli 'indigeni' più illustri (ovvero uno dei membri della classe nobiliare persiana), a testimoniare se non altro la mancanza di sensibilità o duttilità politica di Antigono nei confronti dell'elemento anellenico. ${ }^{67}$

È quindi probabile che nella $\theta v \sigma i ́ \alpha$ di Persepoli abbiano esercitato un ruolo attivo anche i magi, nella disposizione dei cerchi e nella distribuzione delle offerte, per quanto

${ }^{61}$ Sul problema, lungi dal trovare una soluzione definitiva o comunque condivisa, cfr., da ultimi, Wiesehöfer 2007; Wiesehöfer 2010; Engels 2013.

62 Cfr. Callieri 2007, 66-67; Rougemont 2012, 125-127.

${ }^{63}$ Iossif/Lorber 2009, 19-22. D'altro canto, vd. la posizione, fin troppo recisa, di Eckhardt 2015, 272: "On the whole, the Seleucids do not seem to have had any special interest in Achaemenid heritage, and the same is true for Achaemenid religious traditions" (ma senza discutere le tavole di Persepoli).

${ }^{64}$ Wiesehöfer 1994, 53-54, 72-73, che pure ritiene che a Persepoli fossero venerate anche divinità persiane); Wiesehöfer 2007, 39; Wiesehöfer 2010, 109 (cfr. Callieri 2007, 56-57, 66-67; Canepa 2010, 566; Engels 2013, 32-33). Decisamente più prudenti, anche riguardo all'interpretatio, sono Boyce/Grenet 1991, 107; Canali De Rossi 2004, 141-143, nrr. 241-245; Rougemont 2012, 125-129, nrr. $59-63$ (con ampia bibliografia).

65 Il suggerimento è in Altheim 1947, 270, mentre non figura in Altheim/Stiehl 1970, 564-565.

${ }_{66}$ Arr. Anab. 7.23.1 e 3 (cfr. 6.30.3); Diod. 19.14.4; 48.5 (cfr. 19.21.3).

${ }^{67}$ Diod. 19.48.5. La tendenziosità della fonte è evidente anche 48.1-4, dove si afferma che Antigono, giunto in Perside, ricevette onori regali dagli abitanti e fu riconosciuto da tutti signore dell'Asia. La nuova ripartizione delle satrapie riflette la sua ostilità all'elemento iranico. Quella di Perside venne infatti affidata ad Asclepiodoro, nominato, sempre secondo Diodoro, ipparco. 
non siano menzionati da Diodoro. ${ }^{68}$ Questi sono invece citati espressamente nella cerimonia di Opis del 324, dove insieme agli indovini greci diedero inizio alle libagioni che videro coinvolti Alessandro e gli altri partecipanti.

Quello che si profilò a Persepoli fu dunque il tentativo di creare un'unità e coesione tra mondo macedone e mondo persiano, maggiore rispetto agli altri episodi con cui può essere confrontato, e in cui la parte greca è o viene presentata come predominante, visto che tutto ruota intorno alle divinità (greche) e alla coppia Alessandro e Filippo.

Anzi, la cerimonia di Persepoli mal si giustifica, se viene disgiunta dal legame pregresso di Peucesta con Alessandro Magno, fatto valere dallo stesso satrapo di Persia per ottenere il comando supremo nella lotta contro Antigono. ${ }^{69} \mathrm{E}$ dunque, sotto questo aspetto, vi è piena sintonia con l'importanza vitale di Alessandro e della sua memoria nella prima stagione dei diadochi, evidenziata dagli episodi di Babilonia (Tolemeo) e di Cyinda (Eumene), Il tema era sentito ancora fondamentale per i diadochi e considerato strumento propagandistico importante per le truppe, soprattutto quelle macedoni. ${ }^{70}$

Sono comunque problematiche la presenza e l'identificazione del Filippo accanto ad Alessandro, in posizione subordinata se l'ordine riportato da Diodoro è corretto o comunque ha un qualche valore (difficile comunque negarglielo vista la grande precisione nella descrizione). Henkelman pensa, in modo dubitativo, a Filippo Arrideo, ${ }^{71}$ che nella sistemazione cronologica della Biblioteca storica risulta già morto al momento della cerimonia di Persepoli. ${ }^{72}$ La sua legittimità dinastica trova conferma anche nei funerali e nei giochi riservati a lui e alla moglie Euridice, in ossequio al vó $\mu$ o s macedone (già rispettato, in particolare, da Alessandro nei confronti del padre e, successivamente, da Tolemeo I per lo stesso figlio di Filippo). ${ }^{73}$ Inoltre, evidentemente su un piano completamente diverso (un contesto civico), di lui vengono supposte $\tau$ uxí divine a Samo, insieme ad Alessandro IV, sulla scorta della menzione di un agone per i re. ${ }^{74}$ Il richiamo a Filippo Arrideo potrebbe suonare strano, ma forse trova adeguata spiegazione proprio nel collante usato nelle lotte tra i diadochi. Nello scontro tra 'lealisti' e fautori di poteri monocratici potrebbe avere un senso il collegamento con un sovrano legittimo, per quanto negativa sia stata in certi contesti la valutazione sul suo conto. Lo stesso Eumene, come si è visto, sottolineava, sia pur ricorrendo ad un falso epistolare, il rapporto tra Olimpiade e Alessandro IV.

${ }^{68}$ Così Boyce/Grenet 1991, 20 (cfr. 9 e nota 36).

${ }^{69}$ Ciò è evidente in Diod. 19.15.1: Peucesta voleva la supremazia per il gran numero di soldati e per la promozione ottenuta da Alessandro. Il passo va letto in rapporto con 19.14.4-5. Questo aspetto è del tutto eluso nella Vita di Eumene plutarchea, come notava già Berve 1926, II: 319. Cfr. inoltre Diod. 19.56.1-2: Seleuco, rifiugiatosi in Egitto da Tolemeo I, accusò Antigono di aver deposto dalle satrapie e dai posti elevati quanti avevano combattuto con Alessandro, adducendo come esempi le sue vicende personali, l'eliminazione di Pitone e la sottrazione della Perside a Peucesta.

70 Cfr. Errington 1976; Dreyer 2009; Meeus 2009; Wiemer 2015; Alonso Troncoso 2016.

${ }^{71}$ Henkelman 2011, 116.

72 Diod. 19.11.5 (ottobre 317). Sulla data esatta vd. però $A D$ V, p. 7, No. 2 V'12' (25 dicembre 317) e cfr. la 'Cronaca dei diadochi', ora in $B C H P$ 3, 1. 11. 33, 38, con relativo commento.

73 Athen. 4.155a (= Diyll., FGrH 73 F 1, che ricorda anche Cinna, madre di Euridice); Diod. 17.117.4; 18.1.4; 19.52.5; per Filippo II e Alessandro Magno vedi, rispettivamente, Diod. 17.2.1; Iust. 11.2.1 e Paus. 1.6.3. Cfr. Mari 2008; Alonso Troncoso 2009 e, da ultima, Landucci Gattinoni 2015, 138-141 (anche per la vexata quaestio della tomba di Filippo II/Filippo Arrideo a Vergina).

${ }^{74} I G$ 12.6.2.42 (variamente datato tra il 321 e il 319). Cfr. Mari 2004, 184-185 (con la bibliografia ivi citata). Quello di Samo sembra comunque un caso isolato. 
Ma, d'altro canto, non si può negare che Filippo II godesse ancora di un certo prestigio sia tra le truppe ${ }^{75}$ sia anche nella memoria coltivata da Alessandro, per cui la maggior parte della critica, quasi sempre implicitamente, ritiene a ragione che sia proprio lui il personaggio a cui si riferisce Diodoro. Il discorso di Alessandro ai soldati ad Opis nel 324 costituisce elemento interessante a riguardo, nel racconto di Arriano (e parzialmente di Curzio Rufo). Tra gli argomenti evocati per sedare la rivolta vi è anche l'esaltazione di Filippo come sovrano civilizzatore. ${ }^{76}$ Se davvero dunque Peucesta, inserendo le figure di Alessandro e Filippo, si pose in rapporto dialettico con quanto fatto da Eumene, è possibile ritenere che padre e figlio venissero venerati in modo analogo. ${ }^{77} \mathrm{E}$ dunque il richiamo dinastico risulta così rafforzato presso la componente greco-macedone dei contingenti militari, mentre rimane incerto, a mio avviso, quale fosse la ricezione presso la componente iranica (che comunque ben conosceva le preghiere per la fravashi del sovrano defunto). ${ }^{78}$

La presenza di Alessandro e Filippo come paredri delle divinità (cinque, in base alla suggestione epigrafica succitata o forse in numero maggiore?), permette inoltre un confronto con quanto avvenne rispettivamente nel 336 e nel 324, in due episodi ben noti e oggetto di grande discussione critica Nel primo caso, ad Ege nel corso dei festeggiamenti per le nozze della figlia Cleopatra con Alessandro il Molosso, Filippo fece allestire un corteo, portando in processione splendide statue dei dodici dèi e insieme una sua in cui era effigiato come un dio, in modo tale che si presentò assiso sul trono al fianco delle divinità. ${ }^{79}$ D'altro canto, nel contesto della (probabile) richiesta di onori divini del 324 da parte di Alessandro, ad Atene Demade, apertamente filomacedone, era pronto a considerare il Macedone un tredicesimo dio, stigmatizzando l'ostinato attaccamento alla tradizione dei concittadini, e subendo per questo una condanna pubblica dalla comunità formalizzatasi in un processo di empietà ${ }^{80}$ Di un collegamento tra culto dei dodici dèi e di Alessandro vi è una prova a Taso, nell'ultimo quarto del IV secolo, dove sono attestate feste Duodekatheia e Alexandreia, citate le une dopo le altra. ${ }^{81}$

75 Vd. Plut. Eum. 18.2 (sugli Argiraspidi che, di fronte all'imminente disfatta di Eumene di fronte ad Antigono, si lamentano della loro infelice fine, considerandosi i migliori soldati di Filippo e Alessandro); Diod. 19.41.1 (ma con prospettiva rovesciata: viene rinfacciato agli uomini di Antigono di combattere contro i soldati dei due Argeadi).

76 Arr. Anab. 7.9-10, partic. 9.1-6; Curt. Ruf. 10.2.23 (nessun cenno nelle fonti parallele: Diod. 17.109.23; Plut. Alex. 71.1-4; Iust. 12.11.5-8). Sul problema dell'attendibilità dell'assunto (per lo più riconosciuta) e sul rapporto con la storiografia coeva (Tolemeo, Aristobulo, Megastene in primis) cfr., da ultimi, A. Zambrini, in Sisti/Zambrini 2004, 599; Kosmin 2014, 283, nota 95; Squillace 2015, 111-112. Cfr. anche Diod. 17.2.2, su cui cfr. Alonso Troncoso 2009, 279 e lo stesso Philippeum di Olimpia, noto monumento dinastico voluto da Filippo e completato probabilmente solo sotto Alessandro (cfr. Carney 2007).

77 Sulla presenza di Filippo II, cfr. Briant 2008, 209; Mari 2008, 228-230, la quale propone un confronto con Iust. 24.5.9-11 (devozione popolare nei confronti di Alessandro e Filippo II nel 279, all'epoca dello scontro di Tolemeo Cerauno con i Celti).

${ }^{78}$ Questo è aspetto è sottolineato da Olbrycht 2013, 165, per cui si dovrebbe parlare addirittura di "evocations to Alexander and Philip". Possibili, analoghe preghiere da parte di comandanti persiani sono state ipotizzate da Boyce/Grenet 1991, 19.

79 Diod. 16.92.5 e 95.1.

${ }^{80}$ Athen.6.251b; Ael. V.H. 5.12 (ammenda di dieci talenti o addirittura cento; la prima cifra è decisamente più attendibile). Inattendibile è Apsin. Tech. rhet. 9, 470 Walz (su un'analoga proposta di Demade per Filippo II).

${ }^{81}$ SEG 17.415, l. 2, su cui, cfr. Kholod 2016, 502, nota 26. Sul noto rapporto di Alessandro con i dodici dèi, in particolare durante la sua spedizione, vd. le fonti citate e discusse da A. Zambrini, in Sisti/Zambrini $2004,515$. 
Comunque sia, bisogna ritenere che a Persepoli per esplicita volontà di Peucesta venisse praticato un culto, per quanto estemporaneo, tributato congiuntamente ad Alessandro e a Filippo, che si potrebbe definire di tipo divino, anche se completamente diverso è il contesto rispetto a quanto avveniva, in particolare, nei culti di tipo civico conferiti separatamente ai due Argeadi (in vita o post mortem), in particolare al figlio. ${ }^{82}$ Veniva coinvolto l'ambito militare non solo nelle sue più alte sfere (come quello di Cyinda), ma in forma ben più estesa e con importanti elementi di novità (indicata dai $\beta \omega \mu$ oí per gli dèi e i due Argeadi, al posto del trono vuoto e dei regalia di Alessandro). Sia quello di Eumene sia quello di Peucesta, si può ulteriormente osservare, furono esperimenti cultuali molto significativi, a testimonianza di un periodo ricco di fermenti, ma destinati ad essere isolati, in quanto dettati da particolari contingenze. ${ }^{83}$

Gli avvenimenti successivi nella storia di Peucesta sono noti. L'alleanza con Eumene contro Antigono e Seleuco portò alla battaglia di Paretacene, e successivamente a quella decisiva nella Gabiene (316 o 315), dove egli si ritirò di fronte ad Antigono, arrendendosi all'avversario. Il diadoco lo depose e di fatto ne fece un suo sottoposto, con promesse considerate fallaci da Diodoro. ${ }^{84}$

Il personaggio figura in Caria forse tra il 312 e il 310, probabilmente nel contesto della campagna di Antigono in quella regione nel $312 .{ }^{85}$ Come a suo tempo proposto da Momigliano, è lui il Peucesta menzionato nel trattato del dinasta Eupolemo con Theangela e i mercenari ribelli asserragliati in quella città, testo epigrafico di difficile datazione. ${ }^{86}$ È poi menzionato da Filarco assieme a Menelao e Ossitemide, noti philoi di Demetrio Poliorcete, in un contesto databile approssimativamente a dopo la battaglia di Ipso. ${ }^{87}$

Dopo la resa di fronte ad Antigono, la sua figura, dunque, scivolò ben presto dietro le quinte o quasi, giustificando almeno per quel periodo il giudizio che su di lui è stato espresso, quello di essere "a man of no mean ambition". ${ }^{88}$ Ma forse sarebbe opportuno parlare di un sano e prudente realismo, in un'epoca che la tradizione tende a presentare come una lotta senza quartiere per appropriarsi dell'eredità di Alessandro. ${ }^{89}$

82 Cfr., rispettivamente, Mari 2008 e, da ultimo, Kholod 2016. Alessandro è invece associato a Olimpiade in un'iscrizione di un altare di Iasos, assai tarda (I secolo a.C. / I secolo d.C.), il che farebbe pensare a un culto divino; così Maddoli 2015.

${ }^{83}$ Più apparente che reale il confronto, in ambito lagide, con l'esposizione del trono di Alessandro (e di Tolemeo I, espressamente citato, a differenza del primo) nella pompé dei Ptolemaia, così come descritto da Callissino di Rodi (Athen. 5.197-203b, partic. 201c, 202a-b = FGrH $627 \mathrm{~F} 2$ ) ovvero con il trono d'oro di Tolemeo I nel palazzo di Zeus, nei versi di Teocrito (Idyll. 17.16-17). Diversamente, Picard 1954, 8.

${ }^{84}$ Diod. 19.48.5; cfr. 56.1.

${ }^{85}$ Diod. 19.69 e 75 . Per una ricostruzione degli avvenimenti e delle lotte in Asia Minore, cfr. Billows 1990, 119-121.

${ }^{86}$ Cfr. Momigliano 1931 (= Momigliano 1975, 561-562); Robert 1936, 69-86, nr. 52, l. 13; cfr. Diod. 19.75.1-5. Sui contorni storici di Eupolemo e sulla datazione del trattato, cfr., tra gli altri, Billows 1989; Austin 2006, 88-89, nr. 40; Fabiani 2009 (con status quaestionis).

${ }^{87}$ Athen. 14.614e-f (= Phylarch., FGrH 81 F 12). Sulla scorta di $I G 11.4$ 161b, ll. 55, 81; 162b, l. 43, secondo Billows 1990, 418, Peucesta avrebbe accompagnato Demetrio in una campagna nell'Egeo, in un periodo difficilmente definibile.

88 Heckel 1992, 286. Opinione radicalmente contraria in Briant 2008, 210.

89 Vd. Plut. De Alex. Magni fort. aut virt. 2.336e-337a (cfr. 338a, 341a); Pyrrh. 12.3-4, su cui cfr., da ultimo, Strootman 2014. 
BIBLIOGRAFIA

Alonso Troncoso, V. (2009), Some Remarks on the Funerals of the Kings: From Philip II to the Diadochi, in: P. Wheatley, R. Hannah (eds.), Alexander \& His Successors: Essays from the Antipodes, Claremont, CA: 276-298.

Alonso Troncoso, V. (2016), Antigonus Monophthalmus and Alexander's Memory, in: C. Bearzot, F. Landucci (eds.), Alexander's Legacy, Roma: 97-111.

Altheim, F. (1947), Weltgeschichte Asiens in griechischer Zeitalter, vol. I, Halle.

Altheim, F., Stiehl, R. (1970), Geschichte Mittelasiens im Altertum, Berlin.

Anson, E.M. (2015), Eumenes of Cardia: A Greek among Macedonians, $2^{\text {nd }}$ ed., Leiden-Boston.

Austin, M. (2006), The Hellenistic World from Alexander to the Roman Conquest: A Selection of Ancient Sources in Translation, $2^{\text {nd }}$ ed., Cambridge.

Ballesteros Pastor, L. (2011), Xerses redivivus: Mitrídates, rey de Oriente frente a Grecia, in: J.M. Cortés Copete, E. Muñiz Grijalvo, R. Gordillo Hervás (edd.), Grecia ante los Imperios, Sevilla: $253-262$.

Bernhardt, R. (2003), Luxuskritik und Aufwandsbeschränkungen in der griechischen Welt, Stuttgart.

Berve, H. (1926), Das Alexanderreich auf prosopographischer Grundlage, vol. I-II, München.

Billows, R.A. (1989), Anatolian Dynasts: The Case of the Macedonian Eupolemos in Karia, CA 8: 173-206.

Billows, R.A. (1990), Antigonos the One-Eyed and the Creation of the Hellenistic State, Berkeley-Los Angeles-Oxford.

Bosworth, A.B. (1980), Alexander and the Iranians, JHS 100, 1-21.

Bosworth, A.B. (1992), History and Artifice in Plutarch's Eumenes, in: P.A. Stadter (ed.), Plutarch and the Historical Tradition, London-New York: 56-89.

Bosworth, A.B. (2000), Ptolemy and the Will of Alexander, in: A.B. Bosworth, E. Baynahm (eds.), Alexander the Great in Fact and Fiction, Oxford.

Bosworth, A.B. (2002), The Legacy of Alexander: Politics, Warfare, and Propaganda under the Successors, Oxford.

Bosworth, A.B. (2005), Review of Schäfer 2002, Gnomon 77: 684-688.

Boyce, M., Grenet, F. (1991), A History of Zoroastrianism, vol. III, Leiden-New York-KøbenhavnKöln.

Briant, P. (1989), Les Grecs et la décadence perse, in: M.M. Mactoux, E. Geny (éd.), Mélanges Pierre Lévêque, vol. II, Besançon-Paris: 33-47.

Briant, P. (2002), From Cyrus to Alexander: History of the Persian Empire, Winona Lake, IN.

Briant, P. (2008), Lettre ouverte à Alexandre le Grand, Arles.

Brosius, M. (2003), Alexander and the Persians, in: J. Roisman (ed.), Brill's Companion to Alexander the Great, Leiden-Boston: 169-193.

Callieri, P. (2007), L'archéologie du Fars à l'époque hellénistique, Paris.

Callieri, P. (2014), The Cultural Heritage of the Aristocracy of Persis During the Hellenistic Period, in: T. Daryaee, A. Mousavi, K. Rezakhani (eds.), Excavating an Empire: Achaemenid Persia in Longue Durée, Costa Mesa, CA: 88-121.

Calmeyer, P. (1982), Zur Genese altiranischer Motive, VIII: Die »statistische Landcharte des Perserreiches" - I', AMI n. F. 15: 105-187.

Canali De Rossi, F. (2004), Iscrizioni dello Estremo Oriente Greco, Bonn.

Canepa, M.P. (2010), Technologies of Memory in Early Sasanian Iran: Achaemenid Sites and Sasanian Identity, AJA 114: 563-596.

Carney, E. (2007), The Philippeum, Women, \& the Formation of a Dynastic Image, in: W. Heckel, L. Tritle, P. Wheatley (eds.), Alexander's Empire: Formulation to Decay, Claremont, CA: 27-60.

Chrubasik, B. (2016), Kings and Usurpers in the Seleukid Empire, Oxford. 
De Luna, M.E. (2003), La comunicazione linguistica fra alloglotti nel mondo greco. Da Omero a Senofonte, Pisa.

Dreyer, B. (2009), Jeder hat Alexander-Bild, das er verdient: The Changing Perceptions of Alexander in Ancient Historiography, in: P. Wheatley, R. Hannah (eds.), Alexander \& His Successors: Essays from the Antipodes, Cleremont, CA: 56-71.

Eckhardt, B. (2015), Achaemenid Religious Policy after the Seleucid Decline: Case Studies in Political Memory and Near Eastern Dynastic Representations, in: J.M. Silverman, C. Waerzeggers (eds.), Political Memory in and after the Persian Empire, Atlanta: 269-298.

Engels, D. (2013), A New Frataraka Chronology, Latomus 72: 28-80.

Errington, R.M. (1970), From Babylon to Triparadeisos: 323-320 B.C., JHS 90: 49-77.

Errington, R.M. (1976), Alexander in the Hellenistic World, in: E. Badian (éd.), Alexander le Grand. Image et réalité, Genève: 137-179.

Fabiani, R. (2009), Eupolemos Potalou o Eupolemos Simalou? Un nuovo documento da Iasos, EA 42: 61-77.

Geiger, J. (1995), Plutarch on Hellenistic Politics: The Case of Eumenes of Cardia, in: I. Gallo, B. Scardigli (a cura di), Teoria e prassi politica nelle opere di Plutarco, Napoli: 173-185.

Hadley, R.A. (2001), A Possible Lost Source for the Career of Eumenes of Kardia, Historia 50: 3-33.

Hall, J.M. (2002), Hellenicity between Ethnicity and Culture, Chicago-London.

Hamilton, J.R. (1987), Alexander's Iranian Policy, in: W. Will, J. Heinrichs (eds.), Zu Alexander d. Gr. Festschrift G. Wirth, vol. I, Amsterdam: 467-486.

Heckel, W. (1988), The Last Days and Testament of Alexander the Great: A Prosopographic Study, Stuttgart.

Heckel, W. (1992), The Marshals of Alexander's Empire, London-New York.

Heckel, W. (2006), Who's Who in the Age of Alexander the Great: Prosopography of Alexander's Empire, Malden, MA-Oxford-Carlton, Victoria.

Henkelman, W.F.M. (2011), Parnakka's Feast: šip in Pārsa and Elam, in: J. Alvarez-Mon, M.B. Garrison (eds.), Elam and Persia, Winona Lake: 89-167.

Hoover, O.D. (2011), Never Mind the Bullocks: Taurine Imagery as a Multicultural Expression of Royal and Divine Power under Seleukos I Nikator, in: P.P. Iossif, A.S. Chankowski, C.C. Lorber (eds.), More than Men, less than Gods, Studies on Royal Cult and Imperial Worship, Leuven-Paris-Walpole, MA: 197-228.

Hornblower, J. (1981), Hieronymus of Cardia, Oxford.

Iossif, P., Lorber, C.C. (2009), The Cult of Helios in the Seleucid East, Topoi 16: $19-42$.

Kholod, M.M. (2016), The Cults of Alexander the Great in the Greek Cities of Asia Minor, Klio 98: 495-525.

Kosmin, P.J. (2014), The Land of the Elephant Kings: Space, Territory, and Ideology in the Seleucid Empire, Cambridge, MA-London.

Landucci Gattinoni, F. (2004), Introduzione, traduzione e note alla Vita di Eumene', in: C.F. Konrad, F. Landucci Gattinoni, Plutarco. Sertorio - Eumene, Milano: 333-469.

Landucci Gattinoni, F. (2008), Diodoro Siculo. Biblioteca Storica. Libro XVIII. Commento storico, Milano.

Landucci Gattinoni, F. (2011), Diodoro e la cronologia dei diadochi: una storia infinita, in: M. Lombardo, C. Marangio (edd.), Antiquitas. Scritti di storia antica in onore di Salvatore Alessandrì, Galatina: $167-178$.

Landucci Gattinoni, F. (2015), Cult of the Dead and Vision of the Afterlife in Early Hellenistic Macedonia, in: T. Howe, E.E. Garwin, G. Wrightson (eds.), Greece, Macedon and Persia: Studies in Social, Political and Military History in Honour of Waldemar Heckel, Oxford-Philadelphia: $135-142$.

Launey, M. (1950), Recherches sur les armées hellénistiques, vol. II, Paris.

Maddoli, G. (2015), Ara in onore di Alessandro ed Olimpiade, SCO 61: 137-143.

Mari, M. (2004), Il 'culto della personalità' a Samo, tra Lisandro e Demetrio Poliorcete, in: E. Cavallini (a cura di), Samo. Storia, letteratura, scienza, Pisa-Roma: 177-196. 
Mari, M. (2008), The Ruler Cult in Macedonia, Studi Ellenistici 20: 219-268.

Meeus, A. (2009), Alexander's Image in the Age of the Successors, in: W. Heckel, L.A. Tritle (eds.), Alexander the Great: A New History, Malden, MA 2009: 235-250.

Meeus, A. (2013), What We Do Not Know about the Age of the Diadochi: The Methodological Consequences of the Gaps in the Evidence, in: V. Alonso Troncoso (ed.), After Alexander: The Time of the Diadochi (323-281 BC), Oxford: 84-98.

Momigliano, A. (1931), Peucesta, RFIC n.s. 9: 245-246.

Momigliano, A. (1975), Quinto contributo alla storia degli studi classici e del mondo antico, vol. I, Roma.

Muccioli, F. (2004), 'Il Re dell'Asia': ideologia e propaganda da Alessandro Magno a Mitridate VI, Simblos. Scritti di storia antica 4:105-158.

Muccioli, F. (2012), La storia attraverso gli esempi. Protagonisti e interpretazioni del mondo greco in Plutarco, Milano-Udine.

Muccioli, F. (2013), Note a Plutarco. Temistocle, in: AaVv., Plutarco. Temistocle-Camillo, Milano: 224-345.

Muccioli, F. (2016), Classical Sources and proskynesis. History of a Misunderstanding, in: C. Bearzot, F. Landucci (eds.), Alexander's Legacy, Roma: 41-59.

Muccioli, F. (2017), Transferts culturali e cultuali nell'Oriente ellenistico. A proposito di alcuni recenti modelli interpretativi, Sileno, in corso di stampa.

Muccioli, F. (2018a), Ruler Cult and Ancient Biography, PLLS 17, in corso di stampa.

Muccioli, F. (2018b), Lo scontro di Alessandro con i Malli in Plutarco. Realtà storica e deformazione, in: F. Gazzano, G. Traina (éd.), Plutarque et la guerre, Besançon, in corso di stampa.

Olbrycht, M.J. (2013), Iranians in the Diadochi Period, in: V. Alonso Troncoso, E.M. Anson (eds.), After Alexander: The Time of the Diadochi (323-281 BC), Oxford-Oakville 2013: 159-182.

Picard C. (1954), Le trône vide d'Alexandre dans la cérémonie de Cyinda et le culte du trône vide à travers le monde gréco-romain, CArch 7: 1-17.

Pownall, F. (2013), Duris of Samos and the Diadochi, in: V. Alonso Troncoso, E.M. Anson (eds.), After Alexander: The Time of the Diadochi (323-281 BC), Oxford-Oakville, 43-56.

Prandi, L. (2013), Diodoro Siculo. Biblioteca storica. Libro XVII, Commento storico, Milano.

Rathmann, M. (2014), Diodor und seine Quellen. Zur Kompilationstechnik des Historiographen, in: H. Hauben, A. Meeus (eds.), The Age of the Successors and the Creation of the Hellenistic Kingdoms (323-276 B.C.), Leuven: 49-113.

Rathmann, M. (2016), Diodor und seine „Bibliotheke“. Weltgeschichte aus der Provinz, Berlin.

Robert, L. (1936), Collection Frohener, vol. I : Inscriptions grecques, Paris.

Roisman, J. (2010a), Hieronymus of Cardia: Causation and Bias from Alexander to His Successors, in: E. Carney, D. Ogden (eds.), Philip II and Alexander the Great: Father and Son, Lives and Afterlives, Oxford: 135-148, 285-286.

Roisman, J. (2010b), The Silver Shields, Eumenes, and Their Historian, in: A. Erskine, L. Llewellyn-Jones (eds.), Creating a Hellenistic World, Swansea: 61-81.

Roisman, J. (2012), Alexander's Veterans and the Early Wars of the Successors, Austin.

Rougemont, G. (2012), Inscriptions grecques d'Iran et d'Asie centrale, (Corpus Inscriptionum Iranicarum, Part II: Inscriptions of the Seleucid and Parthian Periods and of Eastern Iran and Central Asia, vol. I: Inscriptions in non-iranian languages), London.

Schäfer, C. (2002), Eumenes von Kardia und der Kampf um die Macht im Alexanderreich, Frankfurt am Main.

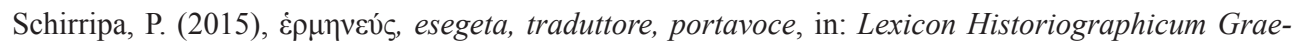
cum et Latinum, vol. 3: $\beta-\zeta$, Pisa: 201-208.

Sisti, F., Zambrini, A. (2004), Arriano. Anabasi di Alessandro, vol. II, Milano.

Squillace, G. (2015), The Comparison between Alexander and Philip: Use and Metamorphosis of an Ideological Theme, in: T. Howe, E.E. Garwin, G. Wrightson (eds.), Greece, Macedon and Persia: Studies in Social, Political and Military History in Honour of Waldemar Heckel, Oxford-Philadelphia: 107-113. 
Strootman, R. (2014), "Men to Whose Rapacity Neither Sea Nor Mountain Sets a Limit". The Aims of the Diadochs, in: H. Hauben, A. Meeus (eds.), The Age of the Successors and the Creation of the Hellenistic Kingdoms (323-276 B.C.), Leuven: 307-322.

Tuplin, C. (2009), The Seleucids and Their Achemenid Predecessors: A Persian Inheritance?, in: S.M.R. Darbandi, A. Zournatzi (eds.), Ancient Greece and Ancient Iran: Cross-Cultural Encounters, Athens: 109-136.

Wiemer, H.-U. (2015), Alexander the Great in the Early Hellenistic Period, in: H. Börm (ed.), Antimonarchic Discourse in Antiquity, Stuttgart: 85-112.

Wiesehöfer, J. (1994), Die ,dunklen Jahrhunderte 'der Persis. Untersuchungen zu Geschichte und Kultur von Fārs in frühhellenistischer Zeit (330-140 v.Ch.), München.

Wiesehöfer, J. (2007), Fars under Seleucid and Parthian Rule, in: V.S. Curtis, S. Stewart (eds.), The Age of the Parthians: The Idea of Iran, vol. II, London-New York: 37-49.

Wiesehöfer, J (2010), Frataraka Rule in Early Seleucid Persia: A New Appraisal, in: A. Erskine, L. Llewellyn-Jones (eds.), Creating a Hellenistic World, Swansea: 107-121. 